\title{
Rare Occurrence of Small-Bowel Volvulus Following Laparoscopic Appendectomy
}

\author{
Andrea Rossetti, MD, Michel Adamina, PD, Franziska Naef, MD, M. Zadnikar, \\ Sascha A. Mueller, PD \\ Klinik für Chirurgie, Kantonsspital St. Gallen, Switzerland (all authors).
}

\begin{abstract}
Introduction: Laparoscopy has proven to be safe for diagnostic and therapeutic purposes in patients with suspected acute appendicitis.

Case Description: We report a case of a rare occurrence of small-bowel volvulus after laparoscopic appendectomy.

Discussion: Moreover, laparoscopy has a number of advantages when compared with open surgery, including faster recovery and shorter length of stay. Nevertheless, laparoscopic appendectomy has a dedicated morbidity. A lifethreatening case of small-bowel volvulus around a properly applied surgical staple line is reported.
\end{abstract}

Key Words: Laparoscopic appendectomy, Laparoscopy, Volvulus.

Citation Rossetti A, Adamina M, Naef F, Zadnikar M, Mueller SA. Rare occurrence of small-bowel volvulus following laparoscopic appendectomy. CRSLS e2014.00162. DOI: 10.4293 /CRSLS.2014.00162.

Copyright (C) 2014 SLS This is an open-access article distributed under the terms of the Creative Commons Attribution-Noncommercial-ShareAlike 3.0 Unported license, which permits unrestricted noncommercial use, distribution, and reproduction in any medium, provided the original author and source are credited.

Address correspondence to: Andrea Rossetti, MD, Kantonsspital St. Gallen, Klinik für Chirurgie, Rorschacherstrasse 95,9007 St. Gallen, Switzerland. Phone: +41 71 49413 12, Fax: +4171 49428 86, E-mail: andrea.rossetti83@gmail.com.

\section{INTRODUCTION}

Laparoscopic appendectomy (LA) has become a standard of care, owing to its superior diagnostic and therapeutic abilities, including faster recovery and shorter length of stay when compared with open appendectomy. LA is also used as an entry procedure in surgical teaching in many institutions, as its safety, ease, and excellent overview are unparalleled. Technical refinements, in particular advanced laparoscopic linear stapling devices, have virtually abolished surgical leaks. ${ }^{1}$ Indeed, surgical complications following LA have become increasingly rare at the population level. ${ }^{2}$ Nonetheless, small-bowel obstruction caused by adhesions to intraperitoneal staples has been reported. ${ }^{3-7}$ Although exceedingly rare, such a complication can be life threatening if not timely recognized and dealt with.

\section{Case Description}

Routine LA was performed in a 30-year-old healthy woman presenting with the typical findings of an acute appendicitis, including an abdominal ultrasonographic scan that was highly suggestive for appendicitis. Standardized 3-trocar diagnostic laparoscopy confirmed the diagnosis. The me- soappendix was dissected using bipolar cautery. The appendix was divided using an endoscopic linear stapler and a $45-\mathrm{mm}$ violet cartridge (Covidien, Boulder, Colorado). No gross intraperitoneal contamination occurred, and the appendix was removed taking advantage of a closed specimen retrieval system. The abdomen was then washed out with $1 \mathrm{~L}$ of warm saline. The procedure was uneventful and lasted 30 minutes with virtually no blood loss. Under the supervision of a consultant surgeon, a surgical resident, who had previously performed $>30$ LAs, performed the procedure. The patient recovered well. She advanced to a normal diet on day 1 and was discharged on postoperative day 2 .

On postoperative day 5 , the patient presented back to the emergency department with severe abdominal pain of acute onset and mild distress. Physical examination revealed a tense abdomen without peritoneal signs. Laboratory findings were reassuring: slight leukocytosis $\left(13.4 \times 10^{9} / \mathrm{L}\right)$ and a C-reactive protein $<3 \mathrm{mg} / \mathrm{L}$. Her vital signs were normal, and she was afebrile. She moved her bowel normally, although she felt nauseous. Abdominal ultrasonography and then a computed tomography scan (Figure 1) revealed fluid-filled, dilated small-bowel loops and free fluid 


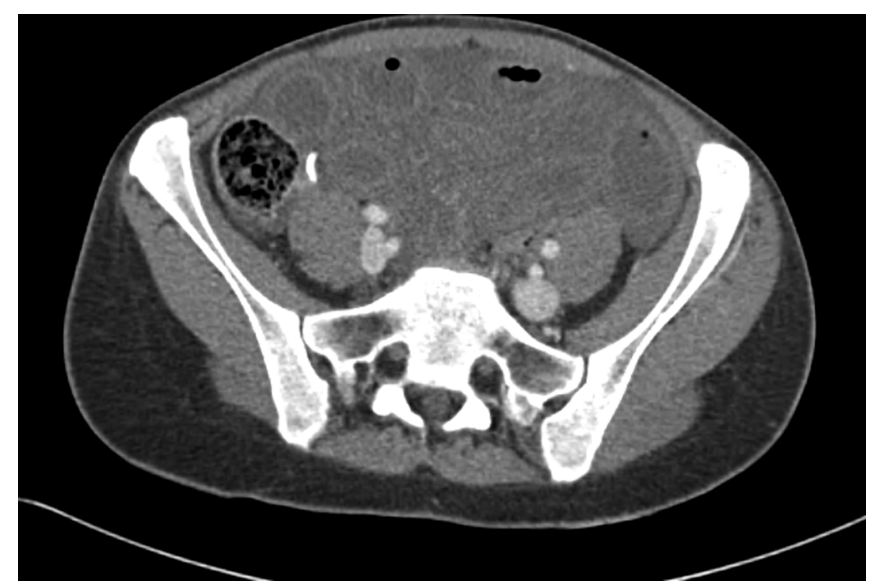

Figure 1. Computed tomography scan showing a small-bowel obstruction with signs of small-bowel ischemia.

in all abdominal quadrants. The patient was readmitted for observation under the working diagnosis of a postoperative ileus. Her condition then deteriorated within hours, and she developed obvious peritoneal signs, thus mandating surgical exploration. Laparotomy over a 10 -cm-long infraumbilical incision was preferred in this sick patient with a now markedly distended abdomen. A 2-m-long segment of ischemic small bowel was encountered, starting $50 \mathrm{~cm}$ distal to the duodenojejunal junction. Bowel occlusion was caused by a $360^{\circ}$ volvulus around an entangled mesentery caught by a partially open surgical staple at the end of an intact staple line (Figures 2 and 3). The cecum and the remainder of the colon appeared normal. Resection of $2.3 \mathrm{~m}$ of the proximal jejunum was required. Bowel continuity was restored with a side-to-side isoperistaltic jejunojejunostomy hand sewn in 2 layers. Thus, the patient was left with $410 \mathrm{~cm}$ of small bowel, including an intact ileum. The laparotomy site was suture closed, and the patient recovered quickly and uneventfully. She was discharged home on postoperative day 7 , and she has remained well since.

\section{DISCUSSION}

Five case reports of small-bowel obstruction following LA were found in a literature review. Of those, 4 obstructions were ascribed to free intraperitoneal staples, ${ }^{3-6}$ and one obstruction was caused by a staple still affixed to the cecum $^{7}$ in a situation similar to that presented here. In one patient, occlusion occurred 3 years after the index appendectomy. ${ }^{6}$ A variety of techniques to close the cecum exists, including using clips, endoloops, and endoscopic staplers, although endoloop and stapler techniques are

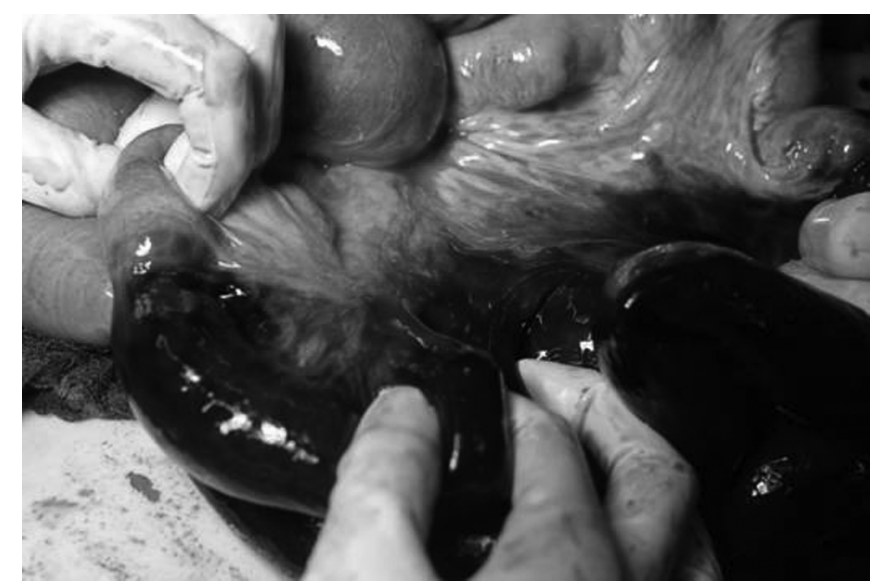

Figure 2. Intraoperative picture revealing small-bowel necrosis due to a volvulus caused by a partially open surgical staple (arrow).

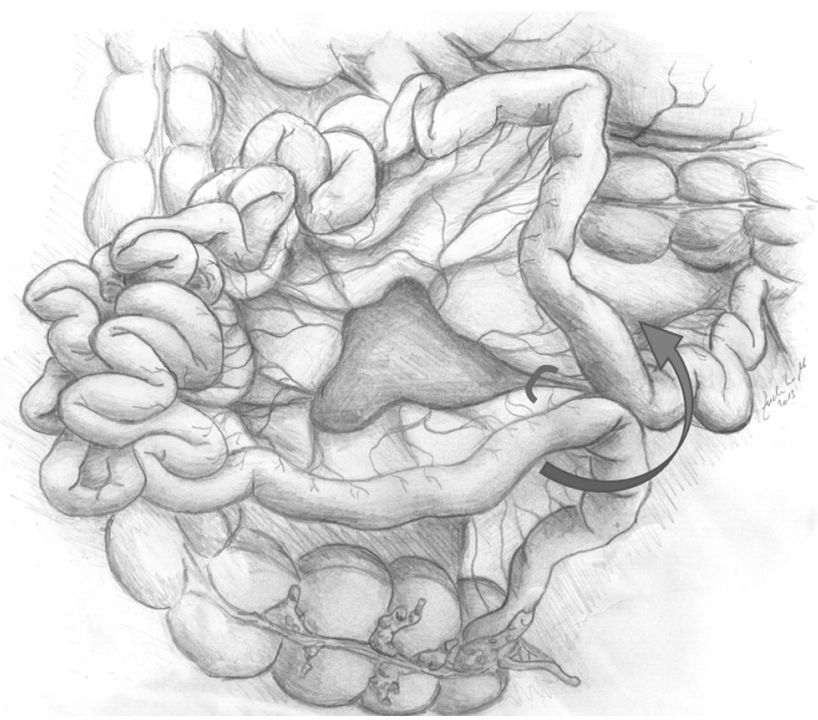

Figure 3. Schema of the small-bowel rotation caused by a surgical staple.

most popular nowadays. A recently published meta-analysis of 5 randomized trials included 622 patients and compared endoloop and endoscopic stapler techniques. Operative time was prolonged when using an endoloop, although this statistical finding was of limited clinical relevance. Total hospital stay was similar between groups. In a fixed-effect meta-analytical model, the endoloop was associated with a higher complication rate; however, this was not observed when repeating the analysis with a random-effect model. Hence, no conclusion can be drawn regarding a potential difference in morbidity. Alternatively, population-based data have convincingly established the safety and effectiveness of an increasing use 
of LA, both with endoloop as with endoscopic stapler techniques. ${ }^{2}$ Also, similar endoscopic staplers are widely used for colorectal resection with minimal technical morbidity associated and proven benefits, in particular when dealing with inflamed tissue. While oversewing a staple line has been advocated as a further mean of reducing potential staple line morbidity, no data sustain such a claim. Contemporary staplers bear a refined staple design to further reduce the likelihood that a staple remains partially open, as happened in the present case with devastating consequences. However, constant vigilance of the surgeon remains the best assurance that a patient recovers well.

This case reminds us of the many pitfalls of surgery. The decision to readmit this patient in spite of reassuring laboratory findings and the swift performance of an exploratory laparotomy within hours of deterioration were crucial to her quick recovery. Resection of a significant amount of small bowel in an otherwise healthy young woman could not be avoided. A small-bowel volvulus around a defective surgical staple is a rare cause of postoperative ileus. Careful inspection and thorough washing during the initial appendectomy revealed nothing remarkable. Nevertheless, a single defective staple was likely overlooked and this was enough to cause a full bowel occlusion. A high index of suspicion at all stages of surgical care remains the best advice for and predictor of a positive outcome.

\section{References:}

1. Sajid MS, Rimple J, Cheek E, Baig MK. Use of endo-GIA versus endo-loop for securing appendicular stump in laparoscopic appendicectomy: a systematic review. Surg Laparosc Endosc Percutan Tech. 2009;19(1):11-15.

2. Brügger L, Rosella L, Candinas D, Güller U. Improving outcomes after laparoscopic appendectomy: a population-based, 12year trend analysis of 7446 patients. Ann Surg. 2011;253(2):309313.

3. Nottingham JM. Mechanical small bowel obstruction from a loose linear cutter staple after laparoscopic appendectomy. Surg Laparosc Endosc Percutan Tech. 2002;12(4):289-290.

4. Kuehnel F, Marusch F, Koch A, Gastinger I. Retained loose linear cutter staples after laparoscopic appendectomy as the cause of mechanical small bowel obstruction. Int J Colorectal Dis. 2007;22(6):717-718.

5. Huntington TR, Klomp GR. Retained staples as a cause of mechanical small-bowel obstruction. Surg Endosc. 1995;9(3):353354 .

6. Chepla KJ, Scott MW. Delayed mechanical small bowel obstruction caused by retained, free, intraperitoneal staple after laparoscopic appendectomy. Surg Laparosc Endosc Percutan Tech. 2011;21:19-20.

7. Petrocelli P, Corsale I, Giannessi S, Cerone M, Colugnat D, Matocci GC. Complications due to mechanical sutures in laparoscopic surgery: bowel obstruction caused by staple: case report and literature review [in Italian]. Minerva Chir. 2003;58(4):591-594. 